\title{
The 'AHR diet' for mucosal homeostasis
}

There is currently much interest in understanding how diet can influence the composition of gut microbial communities and the mucosal immune system. Two independent studies, published in Science and Cell, have identified important roles for diet-derived ligands of the transcription factor aryl hydrocarbon receptor (AHR) in maintaining intestinal immune function.

AHR is expressed by several immune cell types, including retinoic acid receptor-related orphan receptor- $\gamma \mathrm{t}(\mathrm{ROR} \gamma \mathrm{t})^{+}$ innate lymphoid cells (ILCs), which have lymphoid tissue-inducing functions and constitutively produce interleukin-22 (IL-22), and intraepithelial lymphocytes (IELs), which are a distinct population comprising T cell receptor- $\gamma \delta(\mathrm{TCR} \gamma \delta) \mathrm{T}$ cells in the skin and TCR $\gamma \delta$ and CD $8 \alpha \alpha^{+}$ TCR $\alpha \beta$ T cells in the gut. Previous studies have shown that the phytochemical indole-3-carbinol (I3C), which is found in cruciferous vegetables such as broccoli and Brussels sprouts, is an AHR ligand precursor.

Kiss et al. first examined the requirement for AHR in intestinal lymphoid follicle (ILF) organogenesis by generating mice with cell typespecific deletions of Ahr. ILFs were lacking in $A h r^{-/-}$mice and mice with $A h r^{-1-}$ ROR $\gamma \mathrm{t}^{+}$ILCs, but not in mice with $A h r^{-/}$intestinal epithelial cells or dendritic cells. AHR was required for the postnatal expansion of the
ROR $\gamma \mathrm{t}^{+}$ILC pool (but not for the development of these cells), and AHR-mediated expression of the receptor tyrosine kinase KIT was involved in maintaining $\mathrm{ROR} \gamma \mathrm{t}^{+}$ ILC numbers and in ILF formation.

AHR was also found to have a role in IL-22 production by these cells, and IL-22-producing ROR $\gamma \mathrm{t}^{+}$ ILCs were required to maintain the expression of an antimicrobial C-type lectin - regenerating islet-derived protein $3 \gamma$ (REG3 $\gamma)$ - by epithelial cells. Furthermore, $A h r^{-/-}$mice were highly susceptible to intestinal infection with Citrobacter rodentium.

To evaluate a dietary source for AHR ligands, wild-type mice were fed a synthetic diet that lacked ingredients of vegetable origin (synthetic-diet mice). These mice had reduced formation of ILFs and low numbers of ROR $\gamma \mathrm{t}^{+}$ILCs - a similar phenotype to that of $\mathrm{Ahr} \mathrm{r}^{-/-}$mice. Supplementation of this diet with I3C reversed these defects, indicating that plant-derived phytochemicals support the formation of components of the mucosal immune system.

Li et al. focused on the role of AHR in IELs and found that these cells are not maintained in the skin or intestine over time in $A h r^{-1}$ mice or in mice with $A h r^{-/-}$lymphocytes. Turnover of the intestinal epithelial barrier was also reduced in $A h^{-/-}$mice compared with in controls, but transfer of wild-type intestinal TCR $\gamma \delta$ T cells restored epithelial cell proliferation.

\section{restored epithelial cell proliferation.}

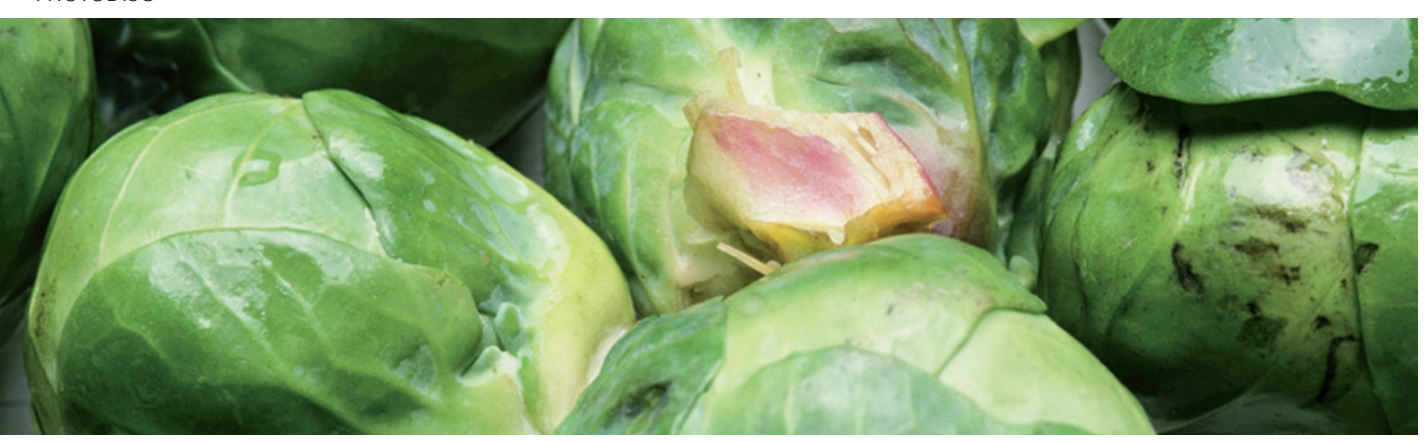

Synthetic-diet mice had a significant reduction in IEL numbers in the intestine. Lower levels of granzymes (which are produced by IELs), matrix metalloproteinase 7, REG3 $\beta$ and REG3 $\gamma$ were detected in the small intestines of $A \mathrm{hr}^{-/}$mice and syntheticdiet mice compared with the levels in control mice. In addition, the bacterial load was increased in the small intestines of $A h r^{--}$mice and synthetic-diet mice, with an increase in the numbers of Bacteroides spp. Supplementation of synthetic-diet mice with $\mathrm{I} 3 \mathrm{C}$ resulted in a bacterial load similar to that of mice fed a standard diet.

Induction of dextran-sulphate sodium (DSS)-mediated colitis in $A h r^{-/-}$mice resulted in more severe disease compared with control mice. A similarly severe disease was observed in DSS-treated syntheticdiet mice, whereas synthetic-diet mice supplemented with I3C showed only mild signs of colitis and recovered rapidly after DSS withdrawal. Transfer of IELs to $\mathrm{Ahr} \mathrm{r}^{-/-}$mice prior to DSS treatment resulted in less severe disease and enhanced recovery following DSS withdrawal. Proliferation of colonic crypt cells was absent in Ahr ${ }^{-1-}$ mice, indicating that activation of AHR by dietary ligands is required to maintain IEL numbers, which help to preserve the epithelial barrier and the microbial load and composition.

These studies are among the first to describe a mechanistic link between dietary compounds and intestinal immune function.

Olive Leavy

ORIGINAL RESEARCH PAPERS Kiss, E. A. et al. Natural aryl hydrocarbon receptor ligands control organogenesis of intestinal lymphoid follicles. Science 27 Oct 2011 (doi:10.1126/ science.1214914) | Li, Y. et al. Exogenous stimuli maintain intraepithelial lymphocytes via aryl hydrocarbon receptor activation. Cell 147 , 629-640 (2011)

FURTHER READING Cheroutre, H., Lambolez, F. \& Mucida, D. The light and dark sides of intestinal intraepithelial lymphocytes. Nature Rev. Immunol. 11, 445-456 (2011) 